\title{
Stochastic volatility models with volatility driven by fractional Brownian motions
}

\author{
T. E. Duncan, J. Jakubowski and B. Pasik-Duncan
}

In this paper the price of a risky asset that has a stochastic volatility being a function of a fractional Brownian motion is considered. Such models can provide a long range dependence for the volatility. The probability density function for the price at a given time is given explicitly under some natural, verifiable conditions. An option pricing model is also considered with some explicit results.

AMS Subject Classifications: 91B25, 91G20, 91G80, 60H30. Keywords AND PHRAses: stochastic volatility, fractional Brownian motion, financial models.

\section{Introduction}

Some research indicates the presence of long range dependence in financial data and in volatility dynamics (e.g. Anderson and Bollerslew [1], Casas and Gao [2], Comte and Renault [4], [5] and Comte et al [3], Ding and Granger [6], Fukasawa [7]). Based on these investigations a market with volatility determined by fractional Brownian motion (FBM) is considered. Specifically it is assumed that the asset price process $X$ satisfies the following stochastic equation

$$
d X(t)=f\left(W^{H}(t)\right) g(t) X(t) d W(t)
$$

where $X(0)$ is a positive constant, the process $W$ is a standard Brownian motion, $W^{H}$ is a standard fractional Brownian motion with Hurst parameter $H \in(0,1), f: \mathbb{R} \rightarrow \mathbb{R}^{+}$is Borel measurable and $g: \mathbb{R} \rightarrow \mathbb{R}^{+}$is Borel measurable and bounded such that the equation (1.1) has a unique strong solution. This approach is motivated by [9]. Following Jakubowski and Wiśniewolski

Research supported by NSF grants DMS 1108884 and DMS 1411412, AFOSR grant FA9550-12-1-0384, ARO grant W911NF-14-10390, Polish MNiSW grant N N201 547838. 
[9] this model (1.1) is called a linear stochastic volatility model with volatility driven by a fractional Brownian motion.

Under some natural assumptions it is verified that the distribution of the asset price $X$ has a probability density function that admits a probabilistic representation (Theorem 2.1).

Subsequently examples of such models are given where the probabilistic representations of the asset price density function is important. The first example is for volatility being a function of a fractional Brownian motion. The second example that is given is the case where the volatility is a geometric fractional Brownian motion.

In Section 3 the probabilistic representations for European call and put option prices in some linear stochastic volatility models are given.

In this paper a similar approach as in Jakubowski and Wiśniewolski [9] is used where the probabilistic representations for the density and European call and put option prices with a linear stochastic volatility model have been given.

\section{The density function of the asset price in a volatility model with volatility given by fractional Brownian motions}

Consider a market defined on a complete probability space $(\Omega, \mathcal{F}, \mathbb{P})$ with filtration $\mathbb{F}=\left(\mathcal{F}_{t}\right)_{t \in[0, T]}, T<\infty$, satisfying the usual conditions and $\mathcal{F}=$ $\mathcal{F}_{T}$. Without loss of generality it is assumed that the savings account is constant and identically equal to one. Moreover, it is assumed that the price $X$ of the underlying asset has a stochastic volatility given by a function of a standard fractional Brownian motion, so the dynamics of $X$ is given by

$$
d X(t)=f\left(W^{H}(t)\right) g(t) X(t) d W(t),
$$

where $X(0)$ is a positive constant, the process $W$ is a standard Brownian motion, $W^{H}$ is a standard fractional Brownian motion with the Hurst parameter $H \in(0,1), f: \mathbb{R} \rightarrow \mathbb{R}^{+}$is Borel measurable and $g:[0, T] \rightarrow \mathbb{R}^{+}$is Borel measurable and bounded. It is well known (e.g. Nualart [10]) that $W^{H}$ has a representation

$$
W^{H}(t)=\int_{0}^{t} K_{H}(t, s) d \widehat{W}(s)
$$


where for $H<1 / 2$

$$
K_{H}(t, s)=c_{H} s^{\frac{1}{2}-H} \int_{s}^{t}(u-s)^{H-\frac{3}{2}} u^{H-\frac{1}{2}} d u,
$$

where $c_{H}=\left[\frac{H(2 H-1)}{\beta\left(2-2 H, H-\frac{1}{2}\right)}\right]^{\frac{1}{2}}, t>s$, and for $H>1 / 2$

$$
\begin{aligned}
K_{H}(t, s)=c_{H}[ & \left(\frac{t}{s}\right)^{H-\frac{1}{2}}(t-s)^{H-\frac{1}{2}} \\
& \left.-\left(H-\frac{1}{2}\right) s^{\frac{1}{2}-H} \int_{s}^{t} u^{H-\frac{3}{2}}(u-s)^{H-\frac{1}{2}} d u\right],
\end{aligned}
$$

where $c_{H}=\left[\frac{2 H}{(1-2 H) \beta\left(1-2 H, H+\frac{1}{2}\right)}\right]^{\frac{1}{2}}, t>s$, and $\widehat{W}$ is a standard Brownian motion. Assume that the stochastic basis is rich enough so that $W$ and $\widehat{W}$ are well defined on it. Let

$$
\int_{0}^{T} f^{2}\left(W^{H}(u)\right) d u<\infty, \quad \mathbb{P}-a . s .
$$

If $f$ is a continuous function, then clearly 2.5 is always satisfied. Since $g$ is bounded, then

$$
\int_{0}^{T} f^{2}\left(W^{H}(u)\right) g^{2}(u) d u<\infty, \quad \mathbb{P}-\text { a.s. }
$$

So, under the assumption 2.5 there exists a unique strong solution of 2.1 and the process $X$ can be expressed as

$$
\begin{aligned}
X(t)=X_{0} \exp ( & \int_{0}^{t} f\left(W^{H}(u)\right) g(u) d W(u) \\
& \left.-\frac{1}{2} \int_{0}^{t} f^{2}\left(W^{H}(u)\right) g^{2}(u) d u\right)
\end{aligned}
$$

(see, e.g., Revuz and Yor [11]). The process $X$ is a local martingale, so there is no arbitrage in the market so defined.

Following 9] this model is called a linear stochastic volatility model, because the stochastic differential equation 2.1 governing the asset price is linear with respect to the asset itself with the coefficient being the stochastic volatility driven by a fractional Brownian motion. 
Now, for a fixed $t$, the probability density function for a stochastic volatility model with volatility given by a function of FBM, which can be correlated with the Brownian motion driving the price equation (2.1) is described.

Theorem 2.1. Let $t \in[0, T], X$ be given by (2.1), $W^{H}$ by (2.2) and $W$, $\widehat{W}$ be correlated Brownian motions, $d\langle W, \widehat{W}\rangle_{t}=\rho(t) d t$ with a measurable, deterministic function $\rho:[0, T] \rightarrow(-1,1)$. Under (2.5) the random variable $X_{t}$ has a probability density function $h_{X_{t}}$ satisfying

$$
\begin{aligned}
& h_{X(t)}(s)=\mathbb{E}\left[\frac { 1 } { s \sigma _ { H } } \varphi \left(\frac{\ln \frac{s}{X_{0}}-\int_{0}^{t} f\left(W^{H}(u)\right) g(u) \rho(u) d \widehat{W}(u)}{\sigma_{H}}\right.\right. \\
&\left.\left.+\frac{\frac{1}{2} \int_{0}^{t} f^{2}\left(W^{H}(u)\right) g^{2}(u) d u}{\sigma_{H}}\right)\right],
\end{aligned}
$$

where $s>0, \varphi$ is the probability density of a standard Gaussian random variable $N(0,1)$, and

$$
\sigma_{H}^{2}=\int_{0}^{t} f^{2}\left(W^{H}(u)\right) g^{2}(u)\left(1-\rho^{2}(u)\right) d u .
$$

Proof. Let $B$ be a standard Brownian motion independent of $\widehat{W}$ such that

$$
W(t)=\rho(t) \widehat{W}(t)+\sqrt{1-\rho^{2}(t)} B(t) .
$$

Let $V$ and $Z$ be defined as follows

$$
\begin{aligned}
Z= & \int_{0}^{t} f\left(W^{H}(u)\right) g(u) \sqrt{\left(1-\rho^{2}(u)\right)} d B(u) \\
& -\frac{1}{2} \int_{0}^{t} f^{2}\left(W^{H}(u)\right) g^{2}(u)\left(1-\rho^{2}(u)\right) d u, \\
V= & \int_{0}^{t} f\left(W^{H}(u)\right) g(u) \rho(u) d \widehat{W}(u) \\
& -\frac{1}{2} \int_{0}^{t} f^{2}\left(W^{H}(u)\right) g^{2}(u) \rho^{2}(u) d u,
\end{aligned}
$$

Then, by (2.7), $Y:=\ln X_{t}=\ln X_{0}+V+Z$. By the independence of $B$ and $\widehat{W}$ it follows that $Z$, conditioned on $\mathcal{F}_{t}^{\widehat{W}}$, has the Gaussian distribution $N\left(-\frac{\sigma_{H}^{2}}{2}, \sigma_{H}^{2}\right)$. So to verify 2.8 it is sufficient to note that the cumulative 
distribution function for $X(t)$ is

$$
\begin{aligned}
\mathbb{P}(X(t) \leq s)=\mathbb{E} \mathbb{E}\left[1_{\left\{Z \leq \ln \frac{s}{X_{0}}-V\right\}} \mid \mathcal{F}_{t}^{\widehat{W}}\right] \\
=\mathbb{E}\left[\Phi \left(\frac{\ln \frac{s}{X_{0}}-\int_{0}^{t} f\left(W^{H}(u)\right) g(u) \rho(u) d \widehat{W}(u)}{\sigma_{H}}\right.\right. \\
\left.\left.+\frac{\frac{1}{2} \int_{0}^{t} f^{2}\left(W^{H}(u)\right) g^{2}(u) d u}{\sigma_{H}}\right)\right],
\end{aligned}
$$

where $\Phi$ denotes the cumulative distribution function of a standard Gaussian random variable. Hence, by the Fubini theorem for nonnegative functions the equality 2.8 follows because

$$
\begin{aligned}
& \frac{\partial}{\partial s} \Phi\left(\frac{\ln \frac{s}{X_{0}}-\int_{0}^{t} f\left(W^{H}(u)\right) g(u) \rho(u) d \widehat{W}(u)+\frac{1}{2} \int_{0}^{t} f^{2}\left(W^{H}(u)\right) g^{2}(u) d u}{\sigma_{H}}\right) \\
&= \frac{1}{s \sigma_{H}} \phi\left(\frac{\ln \frac{s}{X_{0}}-\int_{0}^{t} f\left(W^{H}(u)\right) g(u) \rho(u) d \widehat{W}(u)}{\sigma_{H}}\right. \\
&\left.+\frac{\frac{1}{2} \int_{0}^{t} f^{2}\left(W^{H}(u)\right) g^{2}(u) d u}{\sigma_{H}}\right) .
\end{aligned}
$$

Remark 2.2. If $W$ and $\widehat{W}$ are independent Brownian motions (so $W$ and $W^{H}$ are independent Gaussian processes), then

$$
\sigma_{H}^{2}=\int_{0}^{t} f^{2}\left(W^{H}(u)\right) g^{2}(u) d u
$$

and the equality 2.8 takes a simpler form

$$
h_{X(t)}(u)=\mathbb{E}\left[\frac{1}{u \sigma_{H}} \varphi\left(\frac{\ln \frac{u}{X_{0}}+\sigma_{H}^{2} / 2}{\sigma_{H}}\right)\right],
$$

So to determine the probability density of $X(t)$ it is sufficient to determine the distribution of $\sigma_{H}^{2}=\int_{0}^{t} f^{2}\left(W^{H}(u)\right) g^{2}(u) d u$, provided that 2.5 is satisfied. Since $W^{H}$ is a Gaussian process there are estimation methods to estimate the distribution of $\sigma_{H}^{2}$. 
Remark 2.3. It follows directly that for $H \neq \frac{1}{2}$

$$
\operatorname{corr}\left(W(t), W^{H}(t)\right)=\int_{0}^{t} K_{H}(t, s) d s
$$

where $K_{H}$ is given by $(2.3)$ for $H<1 / 2$, and by $(2.4)$ for $H>1 / 2$.

Theorem 2.1 allows to include two important cases of volatility, the volatility being a function of a fractional Brownian motion and the volatility being a function of a geometric fractional Brownian motion.

Remark 2.4. a) Taking $g \equiv 1$ the problem for volatility being a function of a fractional Brownian motion is solved, that is, $f\left(W^{H}(t)\right)$, so

$$
d X(t)=f\left(W^{H}(t)\right) X(t) d W(t)
$$

provided 2.5 is satisfied.

b) Let the volatility $Y$ be a geometric fractional Brownian motion, that is, $Y$ satisfies the stochastic equation

$$
d Y(t)=Y(t)\left(a d t+b d W^{H}(t)\right)
$$

and $X$ is defined by

$$
d X(t)=Y(t) X(t) d W(t)
$$

Using the form of the unique solution of 2.15 and defining

$$
f(x)=\exp (b x), \quad g(t)=\exp \left(a t-\frac{1}{2} b^{2} t^{2 H}\right)
$$

by Theorem 2.1, the probability density of $X(t)$ is obtained. Indeed, assumption 2.5 is satisfied by continuity of the sample paths.

Example 2.5. If $g \equiv 1, f(x)=|x|$, then 2.5 is satisfied, and in fact the following expectation is easily computed

$$
\mathbb{E}\left[\int_{0}^{t}\left(W_{u}^{H}\right)^{2} d u\right]
$$




\section{Pricing in the model with stochastic volatility driven by a fractional Brownian motion}

Using the probabilistic representation of the density, a closed form of the probability density function is determined in some cases. Initially, recall that $X$ given by 2.1 is a local martingale. If the integrability condition

$$
\mathbb{E}\left(\exp \left(\frac{1}{2} \int_{0}^{T} f^{2}\left(W^{H}(u)\right) g^{2}(u) d u\right)\right)<\infty .
$$

is satisfied then $X$ is a martingale (e.g. [11]).

Note that having the probability density, a formula for the price can be determined for many financial derivatives, indeed from Theorem 2.1 it follows immediately

Corollary 3.1. Let $X$ be the price of an asset with dynamics given by (2.1), and $Y$ be an attainable European contingent claim of the form $Y=F\left(X_{T}\right)$ with maturity at time $T$. If $Y \in L^{2}(P)$ then its price at time 0 is equal to

$$
\int_{0}^{\infty} F(u) h_{X_{T}}(u) d u
$$

where $h_{X_{T}}$ is the density of $X_{T}$.

Therefore for $X$ the closed form solution of prices can be determined and they can be explicitly calculated or numerical methods can be used.

In the next proposition a representation of a vanilla option price is determined. These formulae generalize the famous Black-Scholes formulae as well as a result of Hull and White for a stochastic volatility model with uncorrelated noises [8].

Proposition 3.2. Let $K>0$ be fixed and the price $X$ be given by (2.1). Then

$$
\begin{aligned}
& \mathbb{E}[X(t)-K]^{+}=X_{0} \mathbb{E}\left[e^{V} \Phi\left(d_{1}\right)\right]-K \mathbb{E} \Phi\left(d_{2}\right), \\
& \mathbb{E}[K-X(t)]^{+}=K \mathbb{E} \Phi\left(-d_{2}\right)-X_{0} \mathbb{E}\left[e^{V} \Phi\left(-d_{1}\right)\right],
\end{aligned}
$$

where

$$
d_{1}=\frac{\ln \frac{X_{0}}{K}+V+\frac{\sigma_{H}^{2}}{2}}{\sigma_{H}}, \quad d_{2}=d_{1}-\sigma_{H},
$$

and $\sigma_{H}$ and $V$ are given by (2.9) and 2.12), respectively. 
Proof. Recall that $X(t)=X_{0} \exp (V+Z)$, by (2.7), where $Z$ is given by 2.11). It follows that $V$ is $\mathcal{F}_{t}^{\widehat{W}}$-measurable, so

$$
\mathbb{E}\left(K-X_{t}\right)^{+}=\mathbb{E}\left[X_{0} e^{V} \mathbb{E}\left(\left(\frac{K}{X_{0} e^{V}}-e^{Z}\right)^{+} \mid \mathcal{F}_{t}^{\widehat{W}}\right)\right]:=I .
$$

Since $Z$, conditioned on $\mathcal{F}_{t}^{\widehat{W}}$, has the Gaussian distribution $N\left(-\frac{\sigma_{H}^{2}}{2}, \sigma_{H}^{2}\right)$ (by independence of $B$ and $\widehat{W}$ ) using some classical results it follows that

$$
\begin{aligned}
I & =\mathbb{E}\left[X_{0} e^{V} \frac{K}{X_{0} e^{V}} \Phi\left(\frac{-\ln \frac{X_{0}}{K}-V+\frac{\sigma_{H}^{2}}{2}}{\sigma_{H}}\right)-X_{0} e^{V} \Phi\left(\frac{-\ln \frac{X_{0}}{K}-V-\frac{\sigma_{H}^{2}}{2}}{\sigma_{H}}\right)\right] \\
& =K \mathbb{E} \Phi\left(-d_{2}\right)-X_{0} \mathbb{E}\left[e^{V} \Phi\left(-d_{1}\right)\right] .
\end{aligned}
$$

By the same arguments it follows that

$$
\begin{aligned}
& \mathbb{E}\left(X_{t}-K\right)^{+} \\
= & \mathbb{E}\left[X_{0} e^{V} \mathbb{E}\left(\left(e^{Z}-\frac{K}{X_{0} e^{V}}\right)^{+} \mid \mathcal{F}_{t}^{\widehat{W}}\right)\right] \\
= & \mathbb{E}\left[X_{0} e^{V} \Phi\left(\frac{\ln \frac{X_{0}}{K}+V+\frac{\sigma_{H}^{2}}{2}}{\sigma_{H}}\right)-X_{0} e^{V} \frac{K}{X_{0} e^{V}} \Phi\left(\frac{\ln \frac{X_{0}}{K}+V-\frac{\sigma_{H}^{2}}{2}}{\sigma_{H}}\right)\right] \\
= & X_{0} \mathbb{E}\left[e^{V} \Phi\left(d_{1}\right)\right]-K \mathbb{E} \Phi\left(d_{2}\right) .
\end{aligned}
$$

\section{References}

[1] T. G. Anderson and T. Bollerslew, Heterogeneous information arrivals and return volatility dynamics: Uncovering the long-run in high frequency returns. J. Finance, 52 (1997), 975-1005.

[2] I. Casas and J. Gao, Econometric estimation in long-range dependent volatility models: Theory and practice. J. Econometrics, 147 (2008), 7283.

[3] F. Comte, L. Coutin and E. Renault, Affine fractional stochastic volatility models. Ann. Finance, 8 (2012), 337-378.

[4] F. Comte and E. Renault, Long memory continuous-time models. J. Econometrics, 73 (1996), 101-149. 
[5] F. Comte and E. Renault, Long memory in continuous-time stochastic volatility models. Mathematical Finance, 8 (1998), 291-323.

[6] Z. Ding and C. W. J. Granger, Modelling persistence of speculative returns: A new approach. J. Econometrics, 73 (1996), 185-215.

[7] M. Fukasawa, Asymptotic analysis for stochastic volatility: martingale expansion. Finance and Stochastics, 15 (2011), 635-654.

[8] J. Hull and A. White, The pricing of options on assets with stochastic volatilities. J. Finance, 42 (1987), 281-300.

[9] J. Jakubowski and M. Wiśniewolski, Linear stochastic volatility models. Preprint, arXiv:0909.4765, 2009.

[10] D. Nualart, The Malliavin Calculus and Related Topics (2nd edition). Springer-Verlag, 2006.

[11] D. Revuz and M. Yor, Continuous Martingales and Brownian Motion (3rd edition). Springer-Verlag, 2005.

Department of Mathematics, University of Kansas

LAWRENCE, KS 66045, USA

E-mail address: duncan@ku.edu

Institute of MATHEMATICS, UNIVERSity OF WARSAW

Banacha 2, 02-097 Warszawa, Poland

E-mail address: j.jakubowski@mimuw.edu.pl

Department of Mathematics, University of Kansas

LAWRENCE, KS 66045, USA

E-mail address: bozenna@ku.edu

Received August 17, 2015

Accepted August 27, 2015 
